\section{ESTRATEGIAS DE INTEGRACIÓN URBANA EN CONTEXTOS DE INFORMALIDAD Y AVANCE DE LA INSEGURIDAD'}

Daniela Mariana Gargantini²

\section{Resumen}

La promoción de la integración urbana en sectores urbanos caracterizados por el avance del narcotráfico plantea desafíos particulares, a fin de evitar su expansión y la segregación existente. En coherencia con los postulados del urbanismo social y táctico en pos del mejoramiento y revalorización del espacio público, este artículo indaga sobre las relaciones sinérgicas entre segregación, seguridad, narcotráfico, espacio público y participación ciudadana, a partir de la caracterización y análisis crítico del modelo de intervención integral diseñado en sectores de estas características de la ciudad de Córdoba (Argentina). Bajo este abordaje,

\section{URBAN INTEGRATION STRATEGIES IN CONTEXTS OF INFORMALITY AND THE ADVANCE OF INSECURITY}

Daniela Mariana Gargantini

\section{Abstract}

The promotion of urban integration in sectors characterized by the encroachment of drug trafficking poses particular challenges including avoidance of its expansion and existing segregation. In accordance with hypotheses of social and tactical urbanism and in pursuit of the improvement and revaluation of public space, this paper investigates the synergic relationships between segregation, security, drug trafficking, public space and citizen engagement, based on the characterization and critical analysis of a comprehensive intervention model designed for sectors with these characteristics in the city of Córdoba (Argentina). From this approach, feasible 
se exponen las herramientas de diseño y gestión urbana factibles de ser implementadas en barrios de esta naturaleza, ofreciendo un modelo de intervención posible. Su aplicación articulada a procesos integrales da cuenta de que, frente a la pérdida de valor de los espacios educativos y productivos, las áreas deportivas y para el ocio resultan formas efectivas de avanzar en procesos viables de integración en nuestras urbes. Esto supone una valorización de variables culturales y de la participación de la ciudadanía en la solución de los problemas, a fin de potenciar el desarrollo de mejores políticas urbanas integradoras.

\section{PALABRAS CLAVE: INTEGRACIÓN URBANA, INSEGURIDAD, NARCOTRÁFICO, ESTRATEGIAS DE INTERVENCIÓN}

Recibido: 20-06-2019

Aceptado: 16-10-2019

1 Trabajo producido a partir de los avances y resultados del proyecto "Modelo de intervención integral para la mejora de la calidad de vida de niños y jóvenes que habitan contextos de precarización social de Córdoba capital a partir de la revalorización de espacios públicos" (2017-2018), a cargo de un equipo interdisciplinario de la Universidad Católica de Córdoba (Argentina) bajo financiamiento del Consejo Federal de Inversiones (CFI) de la Nación.

106 revista invi 34(97): 105-127, noviembre 2019 design and management tools to be implemented in neighborhoods with similar situations are presented and offered as a possible intervention model. Its application articulated to integral processes realizes that, in the face of the loss of value of educational and productive spaces, sports and leisure areas are effective ways of advancing in viable processes of integration in our cities. This implies a valorization of cultural variables and the participation of citizens in solving problems, in order to enhance the development of better integrative urban policies.

\section{KEYWORDS: URBAN INTEGRATION, INSECURITY, DRUG TRAFFICKING, INTERVENTION STRATEGIES}

Received: 20-06-2019

Accepted: 16-10-2019

2 Argentina. Investigadora adjunta del Consejo Nacional de Investigaciones Científicas y Técnicas (CONICET); miembro del equipo técnico del Centro Experimental de la Vivienda Económica (Córdoba); docente investigadora de la Universidad Católica de Córdoba, http://orcid.org/0000-0002-0206-2569. Correo electrónico:dmgargantini@gmail.com 


\section{Introducción}

Las ciudades latinoamericanas se han caracterizado históricamente por procesos de desigualdad, creciente fragmentación socioeconómica, y por fuertes tensiones en la construcción y apropiación territorial, fenómenos que han sido problematizados en mayor o menor nivel a lo largo del tiempo.

Las oportunidades de quienes habitan urbanizaciones precarias e informales se encuentran fuertemente condicionadas por variables vinculadas al soporte físico del contexto doméstico y barrial. Si bien la pobreza puede existir aún en contextos donde estas variables físicas se encuentran resueltas, existe una alta correlación entre los déficits del soporte territorial con las zonas de pobreza e informalidad. Variados estudios en la región dan cuenta -con evidencia estadística- de las implicaciones reales de las privaciones en la vida de las personas que habitan estos territorios (Cuenya, 2014; Ferrer y Bagnoli, 2016; Gargantini y Martiarena, 2016; ISSC, IDS y UNESCO, 2016; Marengo y Elorza, 2016).

Desde el aporte de las ciencias sociales -particularmente de la sociología, de la antropología y de la geografía-, hablar de territorio implica considerar la interrelación de los factores biofísicos, humanos y culturales, donde la pertenencia a un territorio se encuentra supeditada a procesos de identificación, subjetivación y representación tanto individual como colectiva (Sack, 1997; Trepat y Cómes, 2000), no necesariamente vinculados a fronteras político-administrativas. De allí su no neutralidad, su dinamismo y su heterogeneidad como iniciativa humana y construcción social, siempre ordenada y organizada por agentes espaciales concretos y generadores de conflicto, en función de intereses y de usos otorgados por cada uno de ellos (Bozzano, 2009; Rodríguez Valbuena, 2010). Los lugares, los procesos y los actores resultan así conceptos fundamentales y organizados en todo estudio territorial y de integración urbana como el que se postula.

Junto con esta consideración, la precariedad urbana y los efectos de segregación que esta genera, resulta uno de los factores condicionantes de la inseguridad en nuestras ciudades (Arriagada Luco y Morales Lazo, 2006).

En materia preventiva, la recuperación de espacios degradados resulta un factor sentido por el urbanismo, al constatarse que, a nivel ciudadano, el escenario más inseguro se relaciona no con las características intrínsecas del lugar personal que se habita, sino con la falta de apropiación del mismo, particularmente del espacio público. La falta de apropiación del espacio público contribuye a la generación de territorios "liberados" donde, entre otros, el problema de la droga agrava el ambiente urbano contemporáneo y favorece tanto la inseguridad como la ejecución de delitos, generándose 
así un círculo de sinergia negativa entre segregación, falta de apropiación de los espacios públicos, liberalización de los mismos, cooptación territorial por parte de grupos asociados al narcotráfico o a actividades ilegales, crecimiento de delitos e incremento real y perceptual de la inseguridad.

Bajo estas consideraciones conceptuales, este trabajo surge a partir de los avances y resultados del proyecto "Modelo de intervención integral para la mejora de la calidad de vida de niños y jóvenes que habitan contextos de precarización social de Córdoba capital a partir de la revalorización de espacios públicos", el cual estuvo a cargo de un equipo interdisciplinario de la Universidad Católica de Córdoba (Argentina) durante los años 2017 y 2018, bajo la solicitud y el financiamiento del Consejo Federal de Inversiones (CFI) de la Nación. El mismo explora como ámbito de recorte territorial el reconocido por los actores que interactúan en barrios Müller y Maldonado de la ciudad de Córdoba (Argentina), identificando a estos sectores como su ámbito de actuación y pertenencia, a partir de la consideración de sus representaciones, identificación de zonas homogéneas, límites y particularidades. Para ello propone la caracterización y análisis crítico del modelo de intervención de integración urbana diseñado y puesto en marcha en esta zona, una de las que registran la más baja calidad de vida, altos grados de informalidad y notable avance de la inseguridad asociada al narcotráfico de Córdoba, segunda ciudad en importancia y escala poblacional de la República Argentina.

Desde los postulados del urbanismo social y táctico, y a partir del reconocimiento de la necesidad de habilitar a cada ciudadano para involucrarse en el proceso creativo de la planificación colectiva, participativa, intra e interactoral, se exponen las herramientas de diseño y gestión implementadas en este sector a fin de sanar el deterioro urbano y propiciar una transformación de estos asentamientos, ofreciendo un método más realista y menos costoso para los planificadores de la ciudad y los ciudadanos, como una forma efectiva de avanzar en una mayor integración urbana como elemento central en la consecución del derecho a la ciudad en nuestras urbes.

Desde este abordaje conceptual y metodológico, el trabajo se estructura a partir de la hipótesis que sostiene que la puesta en funcionamiento de mecanismos integradores más diversos y efectivos relacionados no ya al mundo del trabajo sino al ocio y la recreación, entre los que se encuentran los vinculados a la recuperación de los espacios públicos desde la participación ciudadana, resultan procesos conducentes a incidir en el diseño de políticas públicas innovadoras de integración y seguridad urbana. 


\section{Problemática y estado del arte}

\section{SEGREGACIÓN, ESPACIO PÚBLICO, NARCOTRÁFICO E INSEGURIDAD}

Durante las décadas de los ochenta y noventa, la pobreza -y antes la marginalidad- fueron los centros de atención de los debates sociales y urbanos en América Latina. En ellos, la vivienda, entendida en un concepto amplio e integral, se visualizaba -a partir de la promoción de procesos autogestivos y de inclusión socio-laboral- como una línea estratégica de combate de estos fenómenos.

En las últimas décadas el eje de discusión comenzó a centrarse ya no sólo en la pobreza sino en la segregación urbana y los efectos que esta genera a nivel de su profundización y de la cohesión social de las ciudades. La segregación residencial, entendida como un fenómeno socio-espacial "que atañe a la proximidad territorial (concentración espacial) entre personas pertenecientes a un mismo grupo socioeconómico" (Sabatini, 2004, p. 279), y "como el grado de mixtura presente en la estructura urbana (niveles de homogeneidad o heterogeneidad a nivel de composición social y capacidades urbanas instaladas) desde la consideración de la distancia geográfica entre grupos sociales en su distribución en la ciudad" (Marengo y Elorza, 2016, p. 125), permitió observar y dar cuenta del nivel de jerarquización y más específicamente de desigualdad social y de ventajas relativas gozadas por ciertos sectores a nivel territorial. La interrelación de las dimensiones espaciales y sociales en las conformaciones urbanas cobró así protagonismo en los análisis e intervenciones (Saravi, 2008).

Desde el reconocimiento de un goce diferenciado del derecho a la ciudad según el sector social al que se pertenezca y la ocupación territorial consecuente, comenzó a evidenciarse otro fenómeno asociado a la dualidad urbana presente en las ciudades: el crecimiento exponencial de la violencia producto de la inseguridad ante un otro considerado siempre extraño y potencialmente peligroso. Derivadas de ello, las agresiones cotidianas que se evidencian en nuestras urbes conducen a un ostracismo creciente y a la pérdida de los intercambios sociales, lo cual retroalimenta los procesos de segregación (Cuenya, 2014; Coraggio, 1998; Gargantini, 2008; Marengo y Elorza, 2016).

Así, aun cuando desde mediados de los sesenta las ciudades a nivel internacional comenzaron a experimentar un crecimiento acelerado de la delincuencia, es en las últimas décadas cuando se verifica, progresivamente, que las opciones acerca de dónde vivir, dónde trabajar, dónde matricular a los hijos y dónde comprar, fueron convirtiéndose en decisiones que se toman en consideración a la percepción de delincuencia o inseguridad de los diferentes sectores de la ciudad (Polese y Stren, 2000). 
La segregación resulta así una perspectiva fundamental con la cual ha comenzado a mirarse y estudiarse el circuito de causas y efectos de la delincuencia e inseguridad urbana, a partir de la evaluación ciudadana de la exposición al riesgo que tienen o perciben en su hábitat (Katzman, 2001; Retamoso y Corbo, 2003; Rodríguez, 2001; Sabatini, Cáceres y Cerda, 2001). Junto con ello, es notable el efecto nocivo que esta provoca en la movilidad social y en las perspectivas de inclusión futuras, frente a una creciente tolerancia o indiferencia a estos fenómenos en ciertas zonas excluidas (Arriagada Luco y Morales Lazo, 2006).

Esta delincuencia e inseguridad se encuentran agravadas por el crecimiento del narcotráfico en las principales ciudades de Latinoamérica y el mundo (Klipphan, 2018): segregación, pobreza, "impermeabilidad" o inaccesibilidad urbana, despoblamiento del espacio público y narcotráfico resultan partes de un círculo no virtuoso que se potencia.

En Argentina, informes pertenecientes al último año (Klipphan, 2018) dan cuenta que se consumen en promedio 13.341 kilos de cocaína y 184.991 kilos de marihuana. Solo por la venta ilegal de estos estupefacientes, las bandas mafiosas embolsan 1.080 millones de dólares, o el equivalente al presupuesto en Salud que se destinaba ese año en la provincia de Buenos Aires. Estas cifras sirven para comprender el nivel de lucha que protagonizan las bandas mafiosas en los barrios más postergados para instalar búnkers y kioscos de venta de drogas minorista o al «menudeo». El alquiler de habitaciones como "cocinas de drogas", la comercialización y el simple transporte (servicio de "mulas" protagonizado principalmente por niños aprovechando su imputabilidad ante la ley), resultan en estos barrios actividades mucho más rentables que cualquier otra iniciativa productiva que pueda proponerse.

En Córdoba, un documento de Policía Judicial detalla la existencia de 2.100 bocas de venta narco denunciadas en la provincia, sin que exista prácticamente ningún rincón de la capital cordobesa fuera del mapa (Tolchinsky, 2017), evidenciando una tendencia creciente en las dos últimas décadas. Según datos 2017 provistos por la Secretaría de Políticas Integrales sobre Drogas de la Nación (SEDRONAR), Córdoba está en el tercer puesto en cocaína (con un $2 \%$ ), siete puestos arriba del promedio nacional $(1,5 \%)$. Por otro lado, en cuanto a la inseguridad, según las estadísticas del Ministerio de Seguridad de la Nación, y las cifras de población del INDEC para el año 2017, Córdoba también está en el tercer puesto en cuanto a robos, con 17,3 hechos cada mil habitantes, nueve puestos arriba y casi duplicando el promedio nacional. El Observatorio Provincial de Consumo 
de Sustancias Psicoactivas, en un estudio sobre la realidad de la droga en Córdoba, establecía que este fenómeno es consecuencia de una gran variedad de factores causales, donde la influencia del contexto ambiental y la repercusión en al ámbito social, se han convertido en un problema con dimensiones en la salud pública por las consecuencias en el individuo consumidor, su familia y la comunidad (Secretaría de Prevención de la Drogadicción, 2007).

En esta influencia, la calidad y nivel de apropiación de los espacios públicos resulta central (Uceda Maza, Navarro Pérez y Pérez Cosín, 2014). Cada vez más la percepción de un ambiente seguro recae en estos espacios comunes. Los mismos, negados históricamente por la producción habitacional tradicional que ha privilegiado la promoción de viviendas como meros productos físico-constructivos por sobre procesos urbano-habitacionales integrales y sociales (Gargantini, 2005), hoy resultan estratégicos a la hora de hacer frente a los fenómenos preeminentes de desintegración socio-urbana y crecimiento de la violencia que acontecen en las ciudades.

Estudios sobre satisfacción residencial han evidenciado que

"la experiencia de segregación residencial urbana deriva de la falta de apropiación de espacios públicos por parte de los habitantes y la ausencia de conformación de comunidad en los barrios pobres; que los vecinos y los grupos de población se relacionan y usan el espacio público de manera diferencial, lo que determina una experiencia de seguridad e inseguridad distinta; que los adultos jóvenes jefes de hogar constituyen un grupo que siente un alto grado de temor, lo que matiza el estereotipo de que la juventud es la dueña de las calles y esquinas, en detrimento de las demás edades de vecinos; y que la percepción de inseguridad es una construcción social que alude a temores cotidianos y problemas de cohesión local, que debiera ser objeto central de una política efectiva de recuperación, en particular en barrios de vivienda de interés social" (Arriagada Luco y Morales Lazo, 2006).

La delincuencia resulta así un factor generador de mayor segregación, afectando las preferencias de localización y propiciando mayor confinamiento que se traduce en barrios cerrados y espacios públicos enrejados, que se alejan del ideal de ciudad integrada e integradora, accesible para todos.

\section{EL URBANISMO SOCIAL Y TÁCTICO COMO ESTRATEGIA DE SEGURIDAD CIUDADANA}

Durante la década del noventa comenzaron a surgir iniciativas en la región que revalorizaban el mejoramiento urbano como estrategia de intervención. Programas como el Favela-Barrio en Río de Janeiro (Brasil), la experiencia de Bogotá (Colombia) y el Programa Integral de Mejoramiento de Barrios en diversos países (como en Argentina o como el Programa de Mejoramiento de Barrios 
Subnormales de Medellín-PRIMED) tomaron como ejes de desarrollo innovadores la cualificación de las infraestructuras y servicios en las zonas más marginales de la ciudad, y fueron abriendo camino al concepto de "urbanismo social", a partir de la promoción del desarrollo humano integral y la gobernabilidad democrática.

Más próximos en el tiempo (2004) será la experiencia de Medellín la que -a través de la ejecución de Proyectos Urbanos Integrales (PUI) en territorios caracterizados por altos niveles de pobreza, exclusión y segregación residencial- ponga en centro de la escena la satisfacción de necesidades expresadas en términos de derechos -derecho de todos al espacio público, a un ambiente sano, al libre esparcimiento, a la seguridad, a la educación, a la cultura, entre otros- (Elorza, Alvarado Rodríguez, Balcazar, Morillo y Gamboa, 2019; Montoya, 2014). El plan colombiano logró anular el dominio de las bandas narcos sobre las poblaciones desde la asociación de políticas de mejoramiento urbano, educativas, culturales, turísticas y de seguridad ciudadana, desincentivando la represión. Las intervenciones, a diferencia de las anteriores experiencias, se identificaron por sobresalir en las zonas de intervención a partir del uso de la gran escala, una alta calidad en el diseño, uso de tecnología de punta y aceitados mecanismos de promoción turística y marketing urbano. El modelo Medellín se convirtió así en referencia a nivel regional y mundial, reproduciéndose a varias ciudades ${ }^{3}$.

Complementarios a estas iniciativas, los postulados del microurbanismo, de la acupuntura urbana o del urbanismo táctico, plantearon desde la teoría de ecologismo urbano la revitalización urbana no ya desde la construcción de megaproyectos sino a partir de pequeñas intervenciones que buscan el "reuso adaptativo" de ciertas áreas específicas. Experiencias como las plazas de bolsillo desarrolladas en terrenos baldíos en Chile, Uruguay y República Dominicana como en otros países del mundo resultan buenos ejemplos de estos postulados.

Al revitalizarse estos núcleos inician un efecto dominó con resultados positivos para toda la sociedad de onda mayor. El otorgar la palabra a quienes habitan estos lugares, posibilitándoles su participación en los procesos de planificación de su barrio y de su ciudad resulta otra de las características centrales de estos movimientos.

3 A partir de este modelo, en 2015 el Gobierno de la Provincia de Córdoba lanza el Plan Integral de Seguridad Ciudadana y Prevención del delito "Córdoba se encuentra", el cual aborda de manera integral el problema de la seguridad a partir de la modernización de la institución policial y la transformación urbana con apoyo de programas de Desarrollo Social (Elorza et ál., 2019) 
Más allá de las particularidades de cada aporte, el espacio público ha adquirido un rol de importancia en los procesos de transformación e integración urbana de las zonas marginales e inseguras, como ámbito privilegiado de actuación de las políticas de acceso a un hábitat digno y a la ciudad.

\section{Metodología de caracterización y análisis crítico del modelo de intervención desarrollado}

Desde la adopción de los principios del urbanismo social y táctico, y a partir del reconocimiento de la necesidad de habilitar a cada ciudadano para involucrarse en el proceso creativo de la planificación colectiva, participativa, intra e interactoral, se puso en marcha a finales de 2017 y hasta finales de 2018 el proyecto "Modelo de intervención integral para la mejora de la calidad de vida de niños y jóvenes que habitan contextos de precarización social de Córdoba capital a partir de la revalorización de espacios públicos", desarrollado por un equipo interdisciplinario de la Universidad Católica de Córdoba $^{4}$ bajo solicitud y financiamiento del Consejo Federal de Inversiones de la Nación (CFI).

Su objetivo fue desarrollar e implementar un modelo de intervención integral para la mejora de la calidad de vida de niños y jóvenes que habitan una de las zonas más deprimidas de la ciudad de Córdoba y afectada por el narcotráfico, la exclusión y la pobreza (barrios Müller y Maldonado), a partir de la articulación de iniciativas existentes, de la revalorización de espacios públicos, de la conformación de espacios de fortalecimiento de la ciudadanía y de articulación actoral e intra-inter-gubernamental.

El trabajo se estructuró en tres etapas:

- Etapa 1: Caracterización integral y participativa de la situación socio-física-actoral del territorio.

4 El equipo nucleó a docentes-investigadores de Arquitectura, Urbanismo, Ciencias Químicas, Educación, Psicología, Medicina comunitaria y Epidemiología. Financiación: Consejo Federal de Inversiones. Diciembre 2017-diciembre 2018. El mismo estuvo conformado por: Directora Dra. Arq. Daniela Gargantini; investigadores Arq. Ricardo Sargiotti, Mgtr. Rosario Rollán, Mgtr. Miriam Abascal, Esp. Ivana Schroeder y Lic. Marina Chena; investigadores contratados Mgtr. Miguel Martiarena, Mgtr. Lucas Ruarte, Mgtr. Karina Martín y Esp. Alexis Rossa; tesistas Julieta Calvo, Maria Cerrezuela, Luciana Gaviotto, Mariangeles Gonzalez, Ezequiel Vecchi y Alan Navarro Zalim. 
- Etapa 2: Priorización de los ejes de recuperación urbano-ambiental de los espacios públicos. Vinculación a posibles proyectos de inserción socio-laboral. Articulación con estudio epidemiológico de investigación-acción sobre consumo problemático de sustancias ilegales en la zona. Diseño del modelo de intervención.

- Etapa 3: Implementación preliminar de propuestas de revalorización de los espacios públicos significativos. Incidencia en políticas públicas para la implementación del modelo bajo criterios de articulación actoral e intra-inter-gubernamental.

Junto con ello se realizó el monitoreo y evaluación del proceso.

La metodología adoptada se asentó en un enfoque cualitativo de rescate de percepciones y de capacidades presentes en el territorio, desde el aporte del urbanismo social y táctico. En ello fue estratégico el desarrollo de diagnósticos comunitarios y participativos a partir de la aplicación de metodologías de valorización subjetiva del paisaje (Figura 1) y mapeos sociales.
Las mismas permitieron recoger las visiones particulares de los diferentes actores sociales presentes en el territorio (vecinos, organizaciones sociales y técnicos profesionales provenientes de la universidad y de áreas administrativas del gobierno local y provincial), agrupados en el Consejo de Seguridad Barrial del cuadrante ${ }^{5}$. Esta metodología implicó un abordaje integral e interdisciplinario, a partir del reconocimiento de factores urbanos, paisajísticos, culturales, deportivos, sanitarios, laborales y educativos no formales, a partir de los cuales se favoreció la priorización de las zonas de intervención y la naturaleza de las propuestas de rehabilitación urbana, como una forma efectiva de hacer mejoras de pequeña escala en las áreas seleccionadas, que sirvieran de soporte para procesos sociales de mayor alcance de superación de las condiciones sociales, sanitarias y físicas relevadas.

Las mismas se diseñaron de acuerdo a su mayor relevancia sobre el desenvolvimiento de la vida social de niños y jóvenes de la zona, y a factibilidades técnicas, políticas y económicas existentes.

La priorización de las localizaciones, propuestas e intervenciones específicas se realizó a partir de

5 Bajo el Plan Integral de Seguridad Ciudadana provincial, los Consejos Barriales son espacios de resolución de prioridades de corto, mediano y largo plazo, determinadas democráticamente por los líderes barriales bajo la coordinación de un Promotor Barrial asignado. Los mismos funcionan en articulación con el gabinete interministerial, lo que permite ir resolviendo diversos temas en las comunidades de cada cuadrante. 


\section{FIGURA 1. MAPA DE VALORES PERCEPTUALES DE VECINOS SOBRE LA CALIDAD DE LOS ESPACIOS PÚBLICOS. ROJO: NEGATIVO; VERDE: POSITIVO.}

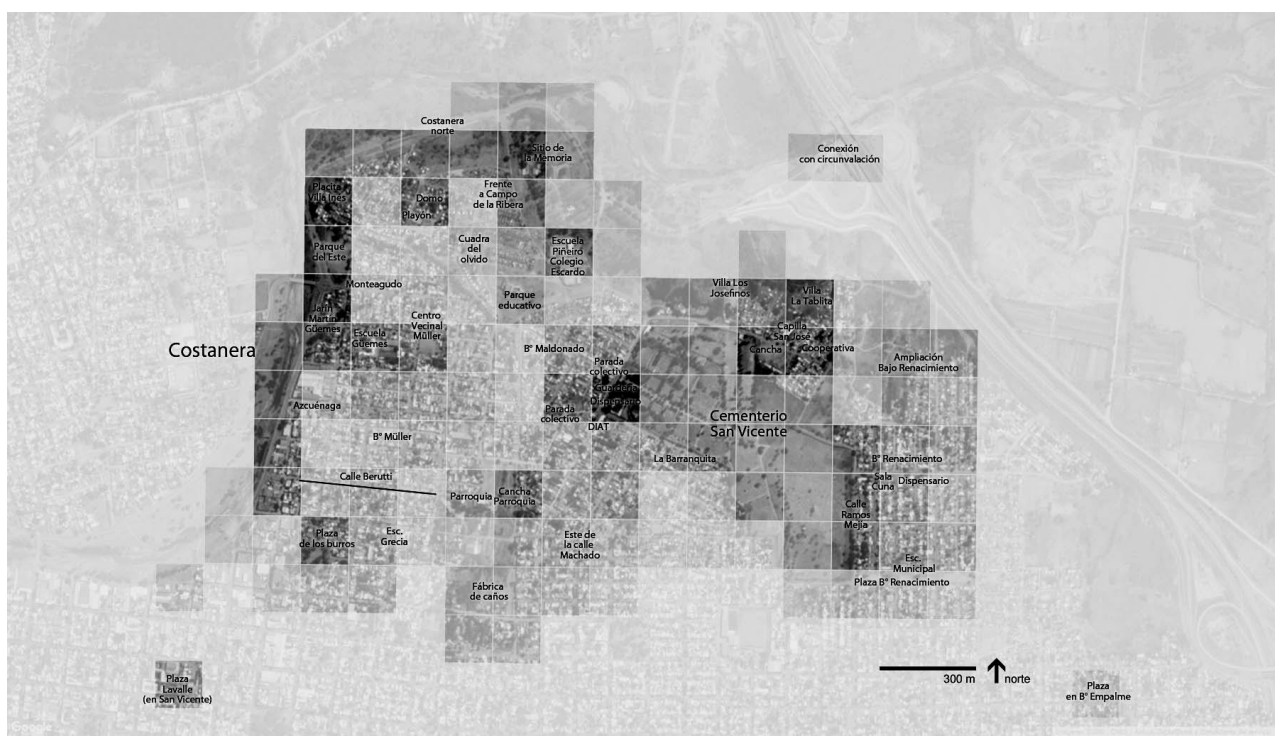

Fuente: Elaborado por Miguel Martiarena, especialista contratado por el proyecto, en Gargantini et ál., 2018

estos espacios de consenso social y del rescate de la apropiación y uso por parte de niños, jóvenes y familias de los espacios existentes, como forma de recuperación ciudadana de zonas cooptadas por el narcotráfico. Como elemento particular de la última etapa del modelo desarrollado, se destacó el involucramiento activo de los actores estatales en estos espacios de consenso, junto a vecinos y el aporte especializado de los investigadores y especialistas que proveyó el proyecto desde la universidad. Una vez obtenida la priorización inicial de vecinos y organizaciones barriales, se procedió a gestionar el vínculo con referentes técnicos y políticos provinciales y municipales (Dirección de Urbanismo de la Secretaría de Desarrollo de Políticas Comunitarias del Ministerio de Seguridad de la Provincia, Dirección de Ambiente de la Municipalidad, Parques educativos, etc.) para conocer 
los proyectos estatales de envergadura planificados en la zona, las posibilidades de que los mismos se sumen en las intervenciones con proyectos, programas o recursos en curso, y las posibles vinculaciones sugeridas con áreas específicas de los diferentes niveles gubernamentales.

De esta manera a las lógicas socio-técnicas articuladas en la primera y segunda etapa, se sumó la lógica política aportando condiciones reales para viabilizar el modelo de intervención y las propuestas de rehabilitación de los espacios públicos sugeridos.

\section{Resultados del modelo de integración urbana desarrollado}

Las propuestas de rehabilitación de espacios públicos, conforme la priorización y validación interactoral realizada (vecinos, referentes comunitarios, técnicos gubernamentales y universitarios), se focalizó en torno a las siguientes estrategias:

- Estrategia 1: El mejoramiento de vías de conexión estructurantes, facilitando la "permeabilidad" de zonas ocultas, inaccesibles y de alta carga negativa de los barrios.

- Estrategia 2: La vinculación de nodos urbanos de uso deportivo-cultural-patrimonial.
En ambos sentidos, la recuperación del eje vertebrador de acceso a la zona más deprimida del sector (Bajada San José) y sus pasajes derivadores, permitieron la conexión urbana con la zona de implantación de las intervenciones de mayor escala en ejecución (nuevo Parque educativo municipal al oeste, mejoramientos en Barrio Renacimiento al sureste, integración de los cementerios y de la costanera, recuperación de inmuebles de valor patrimonial [capilla San José y patio Los Josefinos] junto a zona deportiva anexa) (Figura 2).

Desde estas estrategias se propuso rehabilitar diferencialmente la calle eje (Norberto de la Riestra) de los pasajes transversales. Mientras la calle central se rehabilitó facilitando el ingreso del transporte urbano (extensión de Línea 75 con sus correspondientes paradas), vehicular, sustentable (bicisenda) y peatonal, los pasajes transversales fueron aprovechados como canales depuradores de aguas grises hacia zona baja (inundable y de lagunas) complementando el canal hidrante presente en el sector, y favoreciendo su saneamiento ambiental desde un abordaje urbano-paisajístico y sanitario.

Este eje estructurador priorizado encuentra en su recorrido dos espacios aglutinantes de actividades institucionales-culturales-deportivas-recreativasproductivas con altas potencialidades y factibles de rehabilitar bajo las premisas y el modelo de gestión interactoral propuesto por el proyecto: 
FIGURA 2. ESPACIOS PRIORIZADOS A REHABILITAR A FIN DE FOMENTAR LA INTEGRACIÓN URBANA.

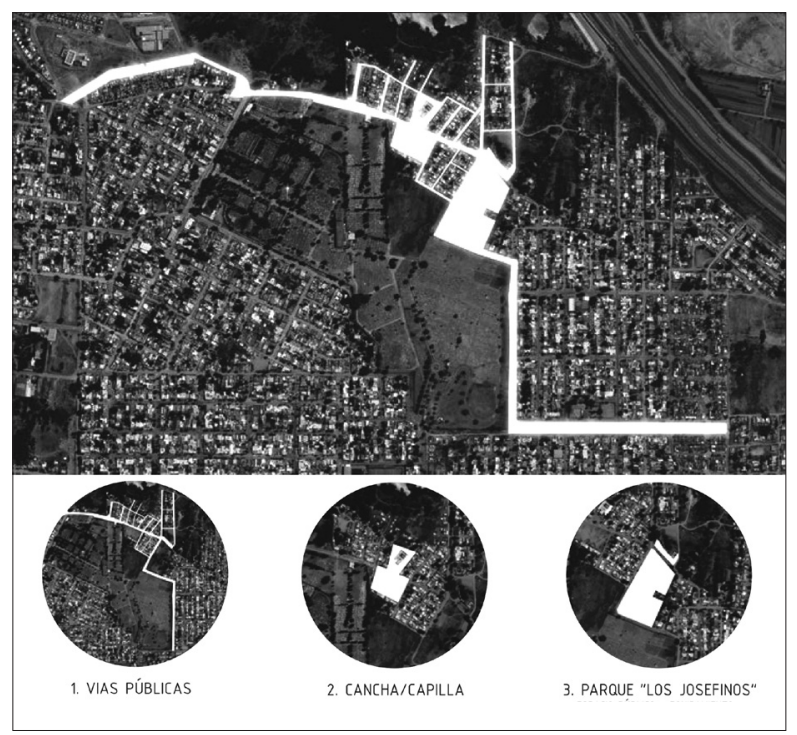

Fuente: Elaboración propia del equipo de proyecto, en Gargantini et ál., 2018.

- La capilla San José, antigua residencia jesuita, su explanada y cancha deportiva ubicada frente a dicha explanada (de dominio municipal).

- Un terreno baldío colindante con ingreso y muro medianero con el cementerio San Vicente, frente a la Cooperativa de reciclados de la zona (de dominio privado), denominado Parque Los Josefinos.

\section{FIGURA 3. PLANIMETRÍA PROPUESTA DE REHABILITACIÓN URBANO-PAISAJÍSTICA EN ZONA CAPILLA Y CANCHA DE FÚTBOL.}

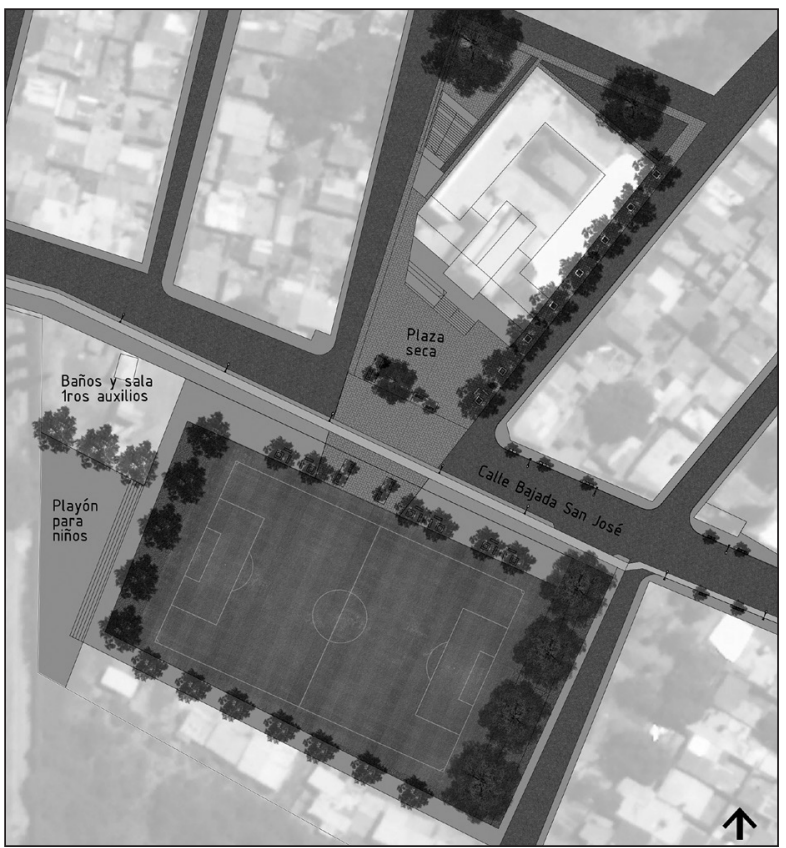

Fuente: Elaboración propia del equipo de proyecto, en Gargantini et ál., 2018.

Bajo el sub-eje de la capilla se planteó rehabilitar en primera instancia su entorno, a fin de capitalizar el alto valor patrimonial de la iglesia (Figura 3). Para ello se planteó una plaza seca que la rodee e incorpore los árboles añosos que la circundan con canteros-bancos. La generación de espacios de 
permanencia en sus inmediaciones resultó uno de los primeros resultados buscados en este sector.

La instalación de escalinatas en el costado oeste de la capilla, de árboles de medio porte en el costado este, así como de rampas y desnivel para la instalación de un escenario frente a la misma, terminó de conformar la propuesta de rehabilitación definida.

El nivel de la plaza seca se continuó sobre la calle, generando una continuidad visual-funcional entre esta y el espacio deportivo propuesto al frente, revalorizando el lugar actualmente apropiado con este fin por niños y jóvenes. La instalación de torres de iluminación, la materialización de los bordes del predio y de la propia cancha de fútbol, constituyeron los aportes centrales del modelo de rehabilitación en el lugar. Los mismos se complementaron con la instalación de arbolado circundante, un playón de juego para niños menores y la instalación de una batería de baños públicos y sala de primeros auxilios en el borde noroeste de la cancha.

Bajo el tercer sub-eje de rehabilitación del Parque Los Josefinos (Figura 4) se propuso completar el modelo de rehabilitación de espacios públicos propiciado por el proyecto. El mismo se basó en la recuperación del gran predio ubicado al frente

\section{FIGURA 4. PLANIMETRÍA PROPUESTA DE REHABILITACIÓN URBANO-PAISAJÍSTICA DE PAROUE LOS JOSEFINOS.}

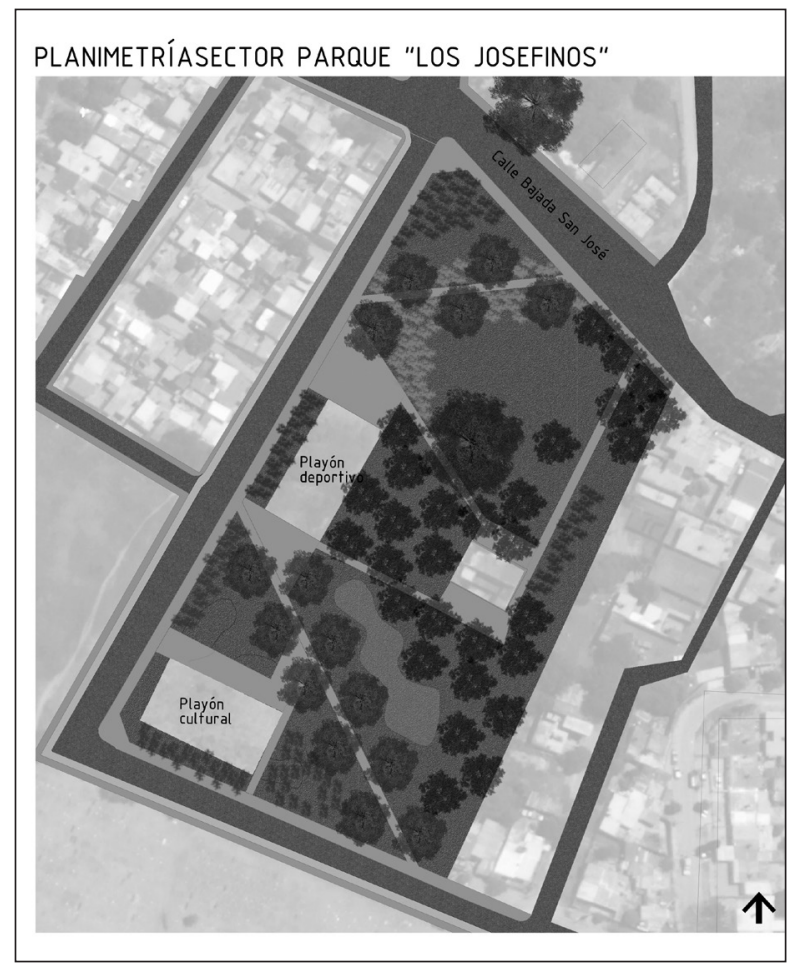

Fuente: Elaboración propia del equipo de proyecto, en Gargantini et ál., 2018. 
de la Cooperativa de reciclado y colindando con el ingreso y muro perimetral del cementerio San Vicente.

Para ello se rescataron los senderos sentidos y ya consolidados de los vecinos en el predio, se propuso reforzar la forestación, incorporar iluminación perimetral e interna, y establecer dos playones: uno con fines deportivos y otro con fines culturales, interconectados entre sí.

La apertura de una calle entre el parque y el cementerio, junto a la intervención y apropiación artístico-popular del muro perimetral del camposanto completó la propuesta de rehabilitación, favoreciendo la circulación vehicular, sustentable y peatonal como estrategia de mejoramiento de las condiciones de seguridad a partir de las microintervenciones urbanas definidas.

- Estrategia 3: La transformación de bordes y límites urbanos de alta carga negativa mediante la intervención artístico-cultural y la recuperación vecinal de las calles.

Desde el eje de la calle central o estructurante se propuso un perfil que transformara el borde impermeable de los muros divisorios de los cementerios (Israelita y católico San Vicente) en un eje de circulación con la incorporación de veredas, canteros hidrantes bajo los cuales se diseñó un sistema de absorción de aguas grises con presencia de plantas nativas, arbolado público de fácil mantenimiento, instalación de murales de construcción colectiva y equipamientos de iluminación, paradas de ómnibus, cestos y puntos de acopio diferencial de residuos. El ensanche de veredas permitió otorgar mayores espacios de permanencia y la dotación de estas cualidades con pequeñas intervenciones de provisión equipamiento urbano buscó favorecer la reapropiación vecinal de las calles, como factor reductor de la inseguridad ante la cooptación de estos espacios por grupos asociados a actividades ilícitas.

- Estrategia 4: La selección de tecnologías favorecedoras de procesos de inclusión laboral juvenil, de oportunidades de capacitación y trabajo frente al crecimiento de actividades ilegales.

Tecnológicamente las obras de mejoramiento se propusieron con sistemas constructivos que requieren baja demanda de mano de obra calificada y favorecen procesos sociales de capacitación y producción autogestionaria de componentes urbanos y habitacionales (ver sistema BENO y MAS en "Tecnologías constructivas", 2015). La producción de componentes urbanos para solados y canteros, así como el cultivo de plantas nativas en un vivero barrial, resultaron dos procesos productivos factibles de favorecer la inclusión socio-laboral de mano de obra femenina y juvenil, integrándolos a la oferta de espacios preventivos, de contención y reinserción ante las adicciones existentes en el barrio. 
Tecnológicamente la intervención se complementó con la recuperación de las actividades de reciclado iniciadas en la Cooperativa (ubicada en el predio frente al Parque Los Josefinos propuesto). Las mismas buscan incluso integrarse al esquema de producción de componentes urbanos a partir de la utilización de ladrillos ecológicos para muros (ladrillos PET), elaborados con una mezcla de partículas de polietileno tereftalato, procedente de envases descartables de bebidas recuperados, ligadas con cemento y aditivos, que se moldea con una máquina manual rodante (ver "Ladrillos de PET", 2015).

- Estrategia 5: Consolidación de espacios comunitarios y de un modo de gestión interactoral.

Junto con ello se reforzó la mesa de gestión conformada por actores referentes del sector (Consejo de Seguridad Barrial), y se propusieron procesos de mejoramiento barrial, de prevención y contención comunitaria, como de inserción socio-productiva de mujeres y jóvenes del lugar.

Este modo de gestión resulta así garante de la sostenibilidad social de las iniciativas propuestas.

En torno a estas estrategias de rehabilitación detalladas se estructuró el modelo de intervención comprometido y se desarrollaron las propuestas de revalorización de los espacios públicos significativos expuestos.

\section{Discusión: Tendencias estratégicas para nuevos procesos de integración urbana}

Los procesos de segregación, producto de una creciente dualidad urbana, plantean nuevos desafíos a las políticas de integración de nuestras ciudades vinculados a la reducción de la inseguridad. Particularmente el caso de estudio seleccionado en este trabajo y el modelo de integración urbana propuesto, dan cuenta de una serie de tendencias estratégicas vinculadas al impulso de nuevas formas de integración urbana en contextos de informalidad y avance de la inseguridad que merecen ser rescatadas. A saber:

- El poder brindar espacios para el ocio, para hacer uso y disfrutar del espacio público, ante la permanencia nula o de corta duración en el espacio público producto de la inseguridad que se vivencia en la zona;

- El potenciar en el territorio "zonas públicas protegidas" por organizaciones del barrio, como respuesta que contrarreste la peligrosidad de los lugares y esquinas problemáticas detectadas; 
- La recuperación de parques de escala (como el Parque del Este existente en la zona o el futuro Parque Los Josefinos) como lugares de potencialidades;

- La apropiación, recuperación y mantenimiento comunitario de los espacios de borde a zonas degradadas (como las murallas de los cementerios católico e israelita existentes y con gran presencia en la zona);

- La modificación-extensión del recorrido del transporte público, y la determinación de una "red de caminos seguros" que facilite el acceso a estos espacios protegidos (tanto de día como de noche);

- La reactivación de las plazas existentes mediante la instalación de ferias, actividades deportivas interbarriales, comerciales y culturales a fin de mejorar la interfase del barrio con el resto de la ciudad;

- El saneamiento de títulos y la potenciación de micro espacios abiertos distribuidas por el barrio;

- El revalorizar las condiciones paisajísticas del lugar (barrancas presentes y vistas privilegiadas que ellas posibilitan);

- La reapertura, mantenimiento y potenciación ambiental-paisajística de vías de conexión y zonas de esparcimiento (como la costanera del río Suquía que constituye el límite norte del sector);
- El empoderamiento e incentivo de espacios públicos para mujeres, a fin de que salgan a la calle y se apropien de los lugares, dado que su presencia es garante de presencia de niños, adolescentes y adultos mayores;

- El brindar soportes físicos para el fomento de actividades que promuevan "que se tomen las calles", especialmente en lugares y horarios considerados peligrosos: murgas, música, procesiones religiosas, carnavales, cine móvil, campeonatos de fútbol interbarriales, etc.

- El vincular la recuperación de espacios públicos con intervenciones que reactiven la economía barrial y generen oportunidades de empleo, dado el bajo nivel educativo y de inserción laboral de la población (como una planta productora de componentes constructivos para el mejoramiento urbano (veredas, bordes, canteros, solados, bancos y mesas premoldeados, etc.), reciclado de basura que se arroja informalmente en la costanera, vivero comunitario destinado a la reforestación de los espacios propuestos, etc.);

- El reforzamiento del trabajo interdisciplinario y en red entre organizaciones presentes en el territorio, así como con organizaciones de barrios vecinos, a fin de ganar impacto e incidencia en las acciones que se encaren; 
- La articulación con entidades responsables del gobierno (en sus distintos niveles (inter-gubernamental) como en sus diversas reparticiones (intra-gubernamental) a fin de garantizar sostenibilidad política y económica a las iniciativas (incidencia en políticas públicas).

Estas tendencias estratégicas guardan consistencia con las perspectivas derivadas del urbanismo social y táctico descripto en el marco teórico elegido, al que se adicionan atributos y exigencias relacionados a la lucha por la descomposición de comunidades urbanas (especialmente juveniles) en manos de actividades delictivas como resulta el narcotráfico.

De esta manera, cobra importancia el desarrollo de programas compensatorios destinados a zonas segregadas y estigmatizadas, que impidan que se cierre el círculo de sinergia negativa compuesto por procesos de segregación e impermeabilidad urbana - no apropiación y liberalización de espacios públicos - cooptación territorial por grupos delictivos y crecimiento de amenazas - incremento de la inseguridad, mediante el aumento de la movilidad, la circulación, la permeabilidad de los territorios, la conexión urbana, y la presencia de nodos estratégicos de encuentro - recuperación regeneración del tejido social y urbano a partir de espacios deportivos, culturales, recreativos y de esparcimiento (Figura 5).
Bajo estas premisas, el mejoramiento y revalorización del espacio público se presenta como un instrumento eficaz para activar otros procesos psico-socio-comunitarios y de diseño-gestión de políticas urbano-habitacionales, dado que su nivel de incidencia tiene el poder de trascender -desde la perspectiva de los sujetos que habitan los territorios- la mera intervención física.

Sin embargo, frente a la tendencia de proyectos monumentales que prometen una modificación o integración a gran escala, y ante la tentación de caer en meros gestos de marketing y competencia urbana bajo criterios y presiones de mercantilización de la ciudad, este tipo de políticas de integración urbana asoma como alternativa para pensar la ciudad proporcionando voz a quienes la habitan y ensayando soluciones a partir de pequeñas intervenciones que buscan transformar la vida cotidiana, sin perder de vista el mayor alcance. Esta lectura "desde abajo" y "desde lo micro", en constante tensión y articulación con lo "macro", junto al reconocimiento de otras voces (disciplinarmente complementarias al urbanismo y cercanas a los actores comunitarios) bajo construcciones de mejoramiento colaborativo, parece resultar más funcional y eficaz frente a los desafíos del contexto actual, a la vez que incentiva una mayor conciencia cívica que se desarrolla a partir de un proyecto inclusivo, social y urbano por medio de micro-proyectos y actividades en torno a estos. 
FIGURA 5. TENDENCIAS ESTRATÉGICAS DE RECONVERSIÓN DEL CÍRCULO DE SINERGIA NEGATIVA PRESENTE EN CONTEXTOS URBANOS INSEGUROS.

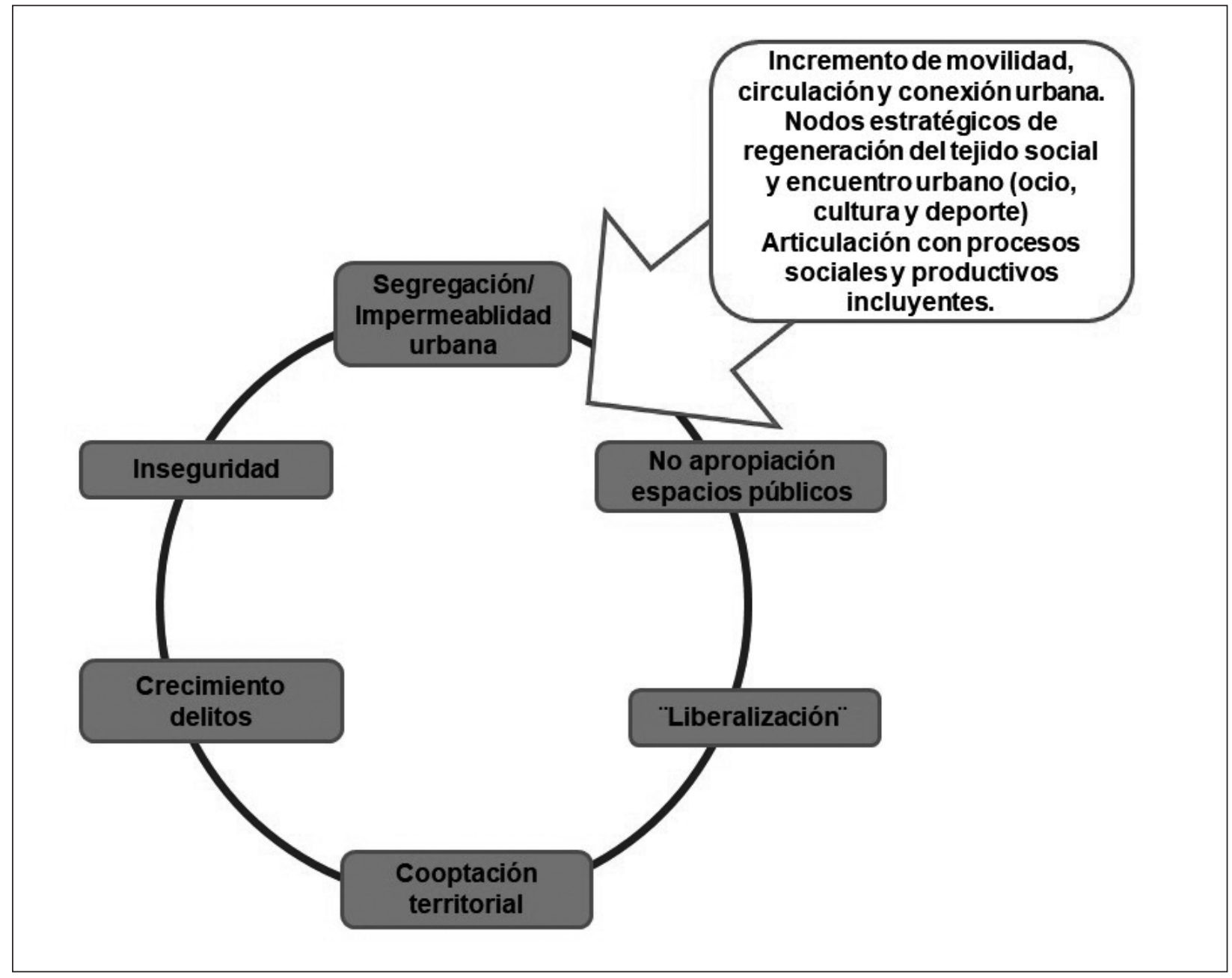

Fuente: Elaboración propia. 


\section{Conclusiones}

Las oportunidades de quienes habitan urbanizaciones precarias se encuentran fuertemente condicionadas por variables vinculadas al soporte físico del contexto doméstico y barrial. En contextos marcados por el avance de actividades delictivas como resulta el narcotráfico, estas variables físicas resultan claves, y la apropiación-rehabilitación-revalorización urbano-ambiental del espacio público resulta indispensable.

Las tendencias estratégicas para alentar nuevos procesos de integración urbana de este tipo de territorios se sustentan en el reconocimiento de que, habiéndose debilitado los ámbitos educativos y el mercado laboral como factores de integración especialmente del sector juvenil, importan la oferta de mecanismos integradores más diversos y efectivos (incluso con capacidad de integración en contextos de inactividad) como resultan el deporte y las actividades vinculadas al ocio y al esparcimiento, contrarrestando así la tendencia de exclusión y estigmatización de estas zonas.

De esta manera el ocio se encuadra dentro de las acciones educativas con capacidad de conquistar el espacio cotidiano de nuestros tiempos, obteniendo un alto nivel de importancia en la planificación de los modelos de ciudad y en las nuevas políticas territoriales de inclusión y promoción socio-educativa.
Frente a las típicas salidas a la violencia delictual urbana vinculadas a la represión, la intervención policial y la privatización del espacio urbano que han dado escasos resultados, estas políticas asumen el control de la misma y el combate de las actividades delictivas desde el repensar, redefinir y fortalecer los espacios de socialización urbana. Esto es recuperar lugares y mecanismos institucionales para la solución de los conflictos, de fomento de una pedagogía para la convivencia, la comunicación y la expresión de sentimientos y habilidades no necesariamente vinculadas a lo productivo. Esto supone una fuerte valorización de las variables culturales y de la participación de la ciudadanía en la solución de los problemas, en articulación con propuestas de concertación con distintas entidades públicas, privadas y comunitarias.

Desde estos postulados, el proyecto aquí descripto promovió el fortalecimiento de procesos de integración urbana a partir de la rehabilitación y el fortalecimiento comunitario desde la perspectiva de los sujetos que habitan los territorios. Con su implementación y sistematización se obtuvieron insumos para el sector y para futuras intervenciones en otras zonas de similares condiciones, en términos de incidencia para el desarrollo de mejores políticas socio-urbano-habitacionales integradoras.

Junto con ello permitió desarrollar y poner en funcionamiento herramientas de diseño y gestión urbana que facilitan sanar el deterioro urbano y 
propiciar una transformación de estos asentamientos, ofreciendo un método más realista, menos costoso y más viable para los planificadores de la ciudad y los ciudadanos, como una forma efectiva de avanzar en la consecución del derecho a la ciudad en nuestras urbes.

\section{Referencias bibliográficas}

Arriagada Luco, C. y Morales Lazo, N. (2006). Ciudad y seguridad ciudadana en Chile: revisión del rol de la segregación sobre la exposición al delito en grandes urbes. Eure, 32(97), 37-48. doi:10.4067/ S0250-71612006000300003

Bozzano, H. (2009). Territorios posibles. Procesos, lugares y actores. Buenos Aires: Lumiere.

Coraggio, J. L. (1998). La gobernabilidad en grandes ciudades: sus condiciones económicas (con especial referencia a la ciudad de Buenos Aires). En Políticas públicas y desarrollo local. Rosario: Instituto de Desarrollo Regional.

Cuenya, B. (2014). Políticas habitacionales inclusivas. En M. Barreto y M. Lentini, Hacia una politica integral del hábitat. Aportes para un observatorio de polí tica habitacional en Argentina (pp. 95-111). Buenos Aires: Café de las Ciudades.

Elorza, A. L., Alvarado Rodríguez, M., Balcazar, F., Morillo, E., y Gamboa, M. (2019, mayo). Estrategias de gestión territorial desde las políticas públicas: replicando el "modelo Medellín" con acento cordobés. En I Encuentro de la Red de Asentamientos Populares: aportes teórico - metodológicos para la reflexión sobre politicas públicas de acceso al hábitat. Encuentro realizado en la Facultad de Arquitectura, Diseño y Urbanismo de la Universidad Nacional de Córdoba, Argentina.

Ferrer,M.yBagnoli,V.(2016).Desafíosurbanosparalaequidad en la infancia. Equidad para la infancia. Recuperado de: http://equidadparalainfancia.org/2016/10/ desafios-urbanos-para-la-equidad-en-la-infancia/

Gargantini, D. (2005). Gestión local del hábitat: experiencias en municipios intermedios. Córdoba: EDUCC Editorial de la Universidad Católica de Córdoba.

Gargantini, D. (2008). La ciudad dual: espacio de libertades restringidas. Córdoba: EDUCC Editorial de la Universidad Católica de Córdoba.

Gargantini, D. y Martiarena, M. (Comps.). (2016). Tierra de conflictos. Conflictos urbanos y violaciones al derecho a la ciudad en Córdoba capital. Córdoba: EDUCC Editorial de la Universidad Católica de Córdoba.

Gargantini, D., Sargiotti, R., Rollán, R., Abascal, M., Schroeder, I., y Chena, M. (2018) Tercer Informe del proyecto modelo de intervención integral para la mejora de la calidad de vida de niños y jóvenes que habitan contextos de precarización social de Córdoba capital a partir de la revalorización de espacios públicos. Córdoba, Argentina: Consejo Federal de Inversiones.

ISSC, IDS y UNESCO. (2016). World social science report 2016. Challenging inequalities: Pathways to a just world. París, Francia: UNESCO. 
Katzman, R. (2001). Seducidos y abandonados: el aislamiento social de los pobres urbanos. Revista de la CEPAL, (75), 171-189.

Klipphan, A. (2018, 31 de marzo). Las alarmantes cifras que mueve el narcotráfico en Argentina para el consumo interno. Diario Clarín. Recuperado de: https://www.infobae.com/sociedad/2018/03/31/ las-alarmantes-cifras-que-mueve-el-narcotraficoen-argentina-para-el-consumo-interno/

Ladrillos de PET. (2015). Centro Experimental de la Vivienda Económica. Recuperado de http://www. ceve.org.ar/materiales-1.php

Marengo, M. C. y Elorza, A. L. (2016). Vivienda social en Córdoba: efectos en la segregación residencial y el crecimiento urbano (1991-2008). Revista INVI, 31(86), 119-144. doi:10.4067/ S0718-83582016000100005

Montoya, N. (2014). Urbanismo social en Medellín: una aproximación desde la utilización estratégica de los derechos. Estudios Políticos, 45, 205-222.

Polese, M. y Stren, R. (Eds.). (2000). The social sustainability of cities: Diversity and the management of change. Toronto: University of Toronto Press.

Retamoso, A. y Corbo, G. (2003). La evolución del sistema urbano uruguayo: una aproximación al fenómeno de la delincuencia y criminalidad en Montevideo. Estados Unidos: Princeton University.

Rodríguez, J. (2001). Segregación residencial socio-económica: ¿qué es? ¿cómo se mide? ¿qué está pasando? ¿importa? Santiago de Chile: CEPAL.
Rodríguez Valbuena, D. (2010). Territorio y territorialidad Nueva categoría de análisis y desarrollo didáctico de la geografía. Revista Uni/PLuri/Diversidad, 10(3).

Sabatini, F. (2004). Medición de la segregación residencial: reflexiones metodológicas desde la ciudad latinoamericana. En F. Sabatini y G. Cáceres (Eds.), Barrios cerrados en Santiago de Chile: entre la exclusión y la integración social. Santiago de Chile: Instituto de Geografía, Pontificia Universidad Católica de Chile.

Sabatini, F., Cáceres, G. y Cerda, J. (2001). Segregación residencial en las principales ciudades chilenas: tendencias de las tres últimas décadas y posibles cursos de acción. Eure, 27(82), 21-42. doi:10.4067/ S0250-71612001008200002

Sack, R. D. (1997). Homo geographicus. A framework for action, awareness and moral concern. London, England: The Johns Hopkins University Press.

Saravi, G. (2008). Mundos aislados: segregación urbana y desigualdad en la ciudad de México. Eure, 34(103), 93-110. doi:10.4067/S0250-71612008000300005

Secretaría de Políticas Integrales sobre Drogas de la Nación Argentina (SEDRONAR). (2017). Estudio nacional en población de 12 a 65 años sobre consumo de sustancias psicoactivas. Argentina, 2017. Principales resultados de la provincia de Córdoba. Recuperado de: http://www.observatorio.gov.ar/media/k2/ attachments/8.3.pdf

Secretaría de Prevención de la Drogadicción (2007). La realidad de la droga en Córdoba. Recopilación de 
Estudios sobre los adolescentes y el consumo de drogas en Córdoba. Córdoba: Observatorio Provincial de Consumo de Sustancias Psicoactivas, Gobierno de la provincia de Córdoba. Recuperado de: http:// archivo.lavoz.com.ar/anexos/Informe/09/8315. pdf

Tecnologías constructivas. (2015). Centro Experimental de la Vivienda Económica. Recuperado de http:// www.ceve.org.ar/tecnologias-constructivas.php

Tolchinsky, F. (2017, 30 de marzo). Revelador: el mapa de las bocas de venta de droga en Córdoba. El Doce. tv. Recuperado de: https://eldoce.tv/sociedad/ revelador-mapa-bocas-venta-droga-cordoba-mirada-telenoche-informe-narcotrafico_38687

Trepat, C. y Cómes, P. (2000). El tiempo y el espacio en la didáctica de las ciencias sociales. Barcelona: Graó.

Uceda Maza, F. X., Navarro Pérez, J. J., y Pérez Cosín, J. V. (2014). El ocio constructivo como estrategia para la integración de adolescentes en conflicto con la ley. Portularia, 14(1), 49-57. doi:10.5218/ prts.2014.0005 